\title{
Metabolic activation of chemical carcinogens by hepatic preparations from streptozotocin-treated rats
}

\author{
P. R. Flatt, S. L. Bass, A. D. Ayrton, J. Trinick and C. Ioannides \\ Biochemistry Department, University of Surrey, Guildford, Surrey, UK
}

\begin{abstract}
Summary. The effect of insulin-dependent diabetes on the hepatic microsomal activation of chemical carcinogens to mutagenic intermediates in the Ames test was investigated in rats pretreated with streptozotocin. In order to discern between the effects of streptozotocin itself and that of the resulting diabetes, groups of streptozotocin-treated rats received either nicotinamide simultaneously with the diabetogenic agent to prevent the onset of diabetes or daily treatment with insulin in order to antagonise the effects of diabetes. The activation of two nitrosamines, nitrosopiperidine and nitrosopyrrolidine was markedly increased following treatment of the animals with streptozotocin, the effect being preventable by nicotinamide and effectively antagonised by insulin. A similar increase in mutagenic response was also seen when Glu-P-1, a carcinogen generated during the cooking of proteinaceous food, was employed as the mutagen. In contrast, the diabetic
\end{abstract}

rats were less efficient than control animals in activating the aromatic amine 2-aminofluorene to mutagenic intermediates. Concomitant administration of nicotinamide with streptozotocin prevented the decrease in mutagenicity, and daily treatment of diabetic rats with insulin partially restored mutagenic response to control levels. Streptozotocin-induced diabetes had no effect on the mutagenicity of 4-aminobiphenyl and the two polycyclic aromatic hydrocarbons, benzo(a)pyrene and 3-methylcholanthrene. The present findings clearly illustrate that diabetes modulates the metabolic activation of carcinogenic chemicals, the effect being dependent on the nature of the carcinogen.

Key words: Metabolic activation, insulin-dependent diabetes, chemical carcinogens, Ames test, aromatic amines, polycyclic aromatic hydrocarbons, food mutagens.
Much of human cancer is believed to result from exposure to chemicals, and numerous examples of chemically-induced carcinogenesis in experimental animals have been documented. The vast majority of these chemicals are per se inactive and require metabolic activation to reactive, electrophilic intermediates that interact with vital cellular macromolecules giving rise to toxicity/carcinogenicity. The major enzyme system responsible for the activation of chemicals is the mixedfunction oxidases, localised on the endoplasmic reticulum of mammalian liver and other tissues. It is primarily concerned with the metabolism of chemicals leading to loss of biological activity, but paradoxically the same enzyme system may direct the metabolism of chemicals towards the formation of toxic/carcinogenic products. It is thus obvious that the extent of formation of a reactive intermediate is largely dependent upon the relative rates of these two competing processes.

The mixed-function oxidase system achieves this dual function, i.e. of activation and deactivation, by existing as a number of structurally and immunologi- cally distinct families of isozymes [1,2]. These families differ in their terminal oxygenase, the haemoprotein cyctochrome P-450, and display markedly different substrate specificities and regio-selectivities [3], so that one family may direct the metabolism of a chemical towards the formation of toxic intermediates while another family may metabolise the same chemical to inert, readily excretable products $[4,5]$. Any factors that alter the ratio of the different families of cytochrome P-450 and thus the balance of activation/deactivation will also interfere with the body's ability to cope with a potential chemical carcinogen.

During the last two decades a number of studies established that induction of insulin-dependent diabetes by streptozotocin (STZ) or alloxan results in marked changes in hepatic mixed function oxidase activity [6-9] which has been attributed to changes in the population of cytochrome P-450 proteins [10-12]. Furthermore, the metabolism of certain carcinogenic nitrosamines, such as $\mathrm{N}$-nitrosodimethylamine and $\mathrm{N}$-nitrosomethylethylamine, was increased in rats rendered diabetic by treat- 

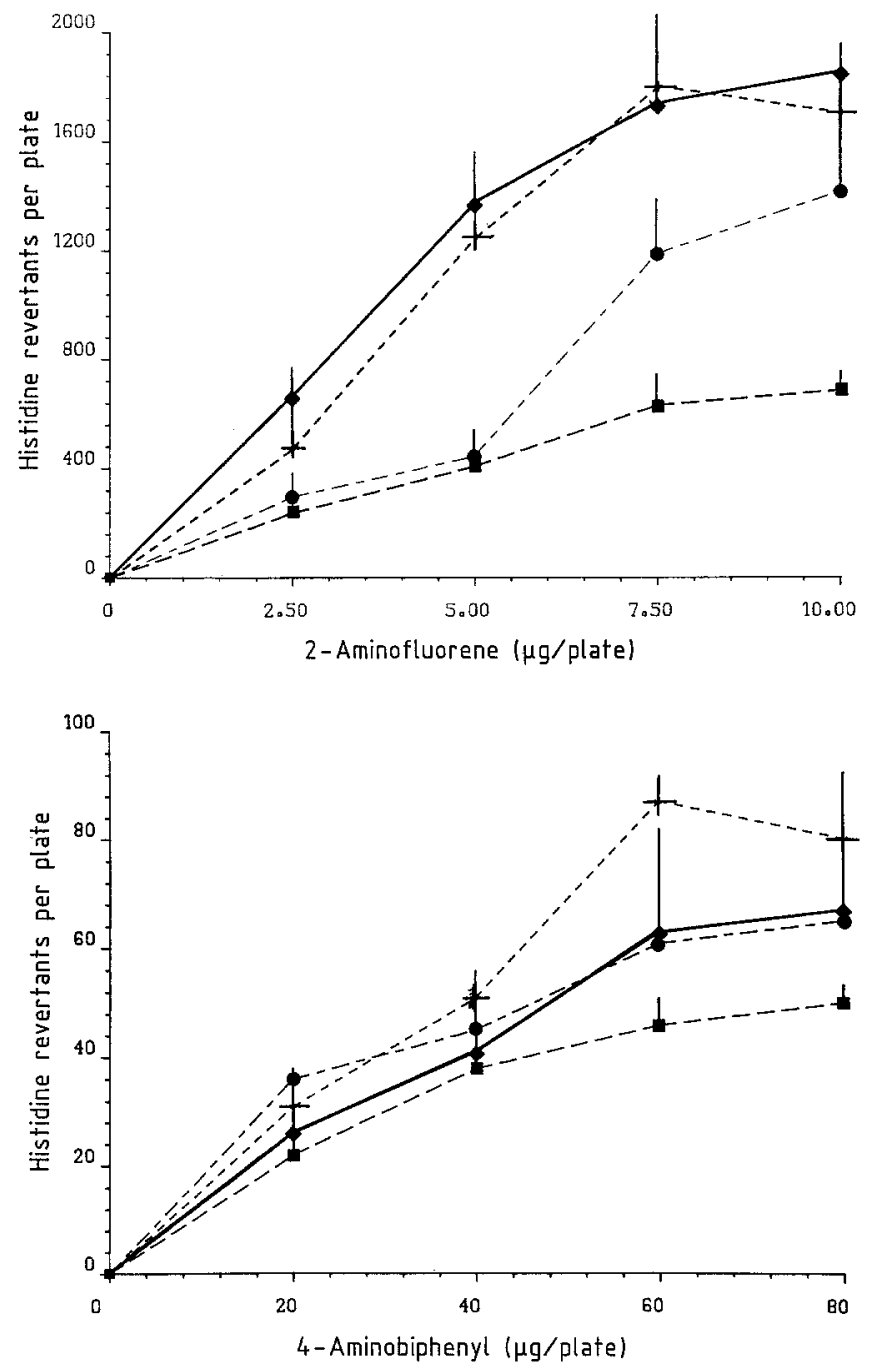

Fig. 1. The metabolic activation of 2-aminofluorene and 4-aminobiophenyl by hepatic preparations derived from streptozotocin-treated rats. The metabolic activation of 2-aminofluorene and 4-aminobiphenyl to mutagens in the Ames test was investigated using the Salmonella typhimurium strain TA 1538 . Activation systems contained $10 \%$ of S9 fraction derived from control $(\bullet)$, streptozotocin $(\boldsymbol{\square})$, streptozotocin + nicotinamide $(+)$ and streptozotocin + insulin $(\bullet)$ treated animals. Results are presented as the mean \pm SD of triplicates. The spontaneous reversion rate of $12 \pm 3$ has already been subtracted. The experiment was repeated with activation systems from different but similarly treated animals with the same results

ment with streptozotocin [13]. Since the cytochrome P450-dependent mixed-function oxidase system plays such a prominent role in the metabolism of many other major groups of chemical carcinogens, the present study was undertaken to investigate the effect of druginduced, insulin-dependent diabetes mellitus on the activation of major groups of established chemical carcinogens, such as aromatic and heterocyclic amines, polycyclic aromatic hydrocarbons and nitrosamines. The generation of reactive intermediates was monitored by the Ames' Salmonella/microsome assay, a test widely used in the detection of potential chemical carcinogens [14].

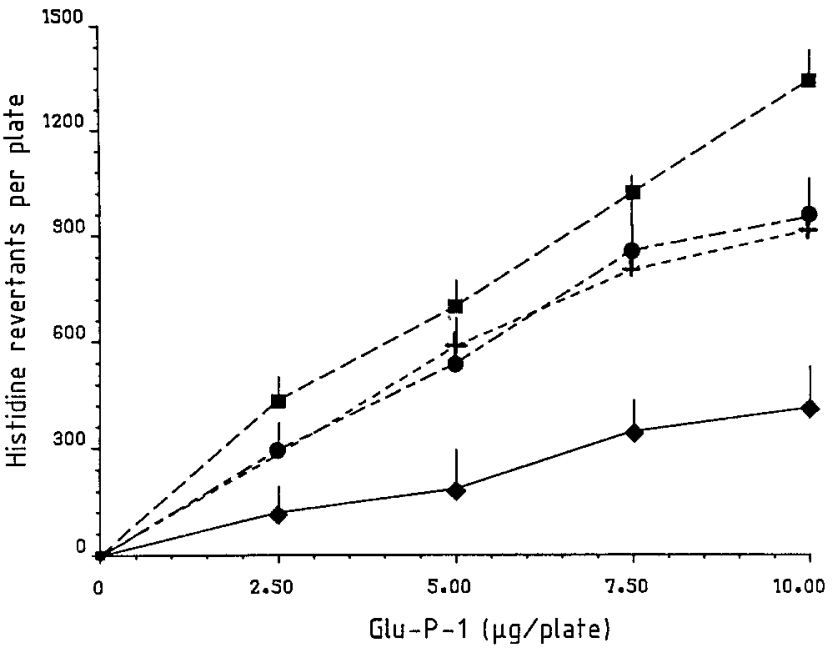

Fig. 2. The bioactivation of Glu-P-1 by hepatic preparations from streptozotocin-treated rats. The metabolic activation of Glu-P-1 to mutagens in the Ames test was investigated using Salmonella typhimurium strain TA 98 . Activation systems contained $10 \%$ of S9 fraction derived from control $(\nabla)$, streptozotocin $(\boldsymbol{\square})$, streptozotocin + nicotinamide $(+)$ and streptozotocin + insulin $(\bullet)$ treated animals. Results are presented as the mean \pm SD of triplicates. The spontaneous reversion rate of $29 \pm 4$ has already been substrated. The experiment was repeated with activation systems from different but similarly treated animals with the same results

\section{Materials and methods}

2-Aminofluorene (Aldrich Chemical Co., Gillingham, Dorset, UK), 4aminobiphenyl (Phase Separations Ltd., Queensferry, Flintshire, UK), Glu-P-1 (2-amino-6-methyldipyrido [1, 2-9:3', 2'-d] imidazole) (Wako Fine Chemicals, Neuss, FRG), and N-nitrosopiperidine, N-nitrosopyrrolidine, benzo(a)pyrene, 3-methylcholanthrene, nicotinamide, streptozotocin and cofactors (Sigma Co., Poole, Dorset, UK) were all purchased. The Salmonella typhimurium strains TA 98, TA 100, TA 1530 and TA 1538 were generous gifts from Professor B. N. Ames, Berkeley, Calif, USA.

Male Wistar albino rats $(180-250 \mathrm{~g})$ were purchased from the Animal Breeding Unit, University of Sulrey, Surrey, UK, and were randomly divided into four groups comprising five animals each. Group A served as the control animals, rats in group B received on day 2 a single intraperitoneal administration of STZ $(65 \mathrm{mg} / \mathrm{kg})$, dissolved in $0.5 \mathrm{~mol} / 1$ sodium citrate buffer, $\mathrm{pH} 4.5$, while rats in group $\mathrm{C}$ received, in addition to $\mathrm{STZ}$, two intraperitoneal doses of nicotinamide $(350 \mathrm{mg} / \mathrm{kg})$, dissolved in $0.9 \% \mathrm{NaCl}, 10 \mathrm{~min}$ before and $3 \mathrm{~h}$ after the STZ. Finally, rats in group D were also treated with STZ, but they additionally received daily subcutaneous doses of longacting monocomponent human insulin (Ultratard; Novo Industrias, Copenhagen, Denmark) as follows: on day 7, three units/rat, days 8-12 five units/rat, increased to 7.5 units/rat on day 13 . The rats were maintained on $10 \mathrm{units} / \mathrm{rat} /$ day from day 14 until the end of the study. All animals were killed by cervical dislocation on day 23, and aliquots of plasma were stored at $-20^{\circ} \mathrm{C}$ for subsequent glucose determination [15].

Hepatic post-mitochondrial (S9) fractions were prepared as previously described [16]. Metabolic activation of the chemical carcinogens by the various hepatic preparations was determined using the Ames' mutagenicity test [13] and employing Salmonella typhimurium strains TA 98, TA 100, TA 1530 and TA 1538. Fresh cultures were prepared by inoculating nutrient broth and incubating overnight at $37^{\circ} \mathrm{C}$ in a shaking water bath. All carcinogens, with the exception of nitrosopiperidine which was dissolved in water, were dissolved in dimethyl sulphoxide so that the amount never exceeded $100 \mu 1$ per plate. The S9 mixes contained $10 \%$ of the hepatic S9 fraction except in the case 
Table 1. Metabolic activation of benzo(a)pyrene and 3-methylcholanthrene by hepatic preparations from diabetic rats

\begin{tabular}{|c|c|c|c|c|c|}
\hline \multirow[t]{3}{*}{ Carcinogen } & \multirow{3}{*}{$\begin{array}{l}\text { Concen- } \\
\text { tration } \\
(\mu \mathrm{g} / \text { plate })\end{array}$} & \multicolumn{4}{|c|}{ Histidine revertants/plate } \\
\hline & & \multicolumn{4}{|c|}{ Animal group } \\
\hline & & A & $\mathrm{B}$ & $\mathrm{C}$ & $\mathrm{D}$ \\
\hline \multirow{5}{*}{$\begin{array}{l}\text { Benzo(a)py- } \\
\text { rene }\end{array}$} & 0 & $77 \pm 2$ & $92 \pm 4$ & $83 \pm 9$ & $76 \pm 10$ \\
\hline & 25 & $92 \pm 6$ & $120 \pm 24$ & $95 \pm 6$ & $76 \pm 10$ \\
\hline & 50 & $93 \pm 7$ & $130 \pm 9$ & $94 \pm 5$ & $86 \pm 5$ \\
\hline & 75 & $119 \pm 16$ & $103 \pm 18$ & $101 \pm 8$ & $84 \pm 8$ \\
\hline & 100 & $107 \pm 11$ & $88 \pm 11$ & $108 \pm 12$ & $89 \pm 3$ \\
\hline \multirow{5}{*}{$\begin{array}{l}\text { 3-Methyl- } \\
\text { cholanthrene }\end{array}$} & 0 & $77 \pm 1$ & $94 \pm 2$ & $79 \pm 4$ & $76 \pm 5$ \\
\hline & 25 & $127 \pm 4$ & $115 \pm 5$ & $140 \pm 8$ & $104 \pm 6$ \\
\hline & 50 & $132 \pm 7$ & $149 \pm 8$ & $176 \pm 21$ & $125 \pm 7$ \\
\hline & 75 & $141 \pm 5$ & $156 \pm 7$ & $166 \pm 7$ & $145 \pm 6$ \\
\hline & 100 & $163 \pm 12$ & $161 \pm 10$ & $184 \pm 14$ & $167 \pm 2$ \\
\hline
\end{tabular}

Results are presented as mean \pm SD of triplicates. Tests were carried out using Salmonella typhimurium strain TA100 and employing 10\% activation systems. Treatment of the various animal groups is described in "Materials and methods"

of the nitrosamines when it was increased to $25 \%$ being equivalent to 0.3 and $0.75 \mathrm{mg}$ of microsomal protein respectively. Activation system, carcinogen and bacteria were always preincubated for $60 \mathrm{~min}$ at $37^{\circ} \mathrm{C}$ in a shaking water bath. A mutagenic response was considered positive only when there was at least a doubling of the spontaneous reversion rate and a concentration-dependent increase in the number of histidine revertants.

\section{Statistical analysis}

Statistical evaluation was carried out using the Student's t-test.

\section{Results}

Rats treated with STZ alone exhibited markedly raised $(p<0.01)$ plasma glucose concentrations $(19.7 \pm$ $4.3 \mathrm{mmol} / 1$; mean $\pm \mathrm{SEM}$ ) compared with either control rats $(5.6 \pm 0.6 \mathrm{mmol} / \mathrm{l})$ or STZ-treated rats given nicotinamide $(7.7 \pm 0.4 \mathrm{mmol} / \mathrm{l})$ or daily insulin injections $(9.0 \pm 1.1 \mathrm{mmol} / 1)$. Plasma glucose concentrations at the time of death of the two latter groups were slightly raised $(p<0.05)$ compared with control rats.

The effect of STZ-induced diabetes on the activation of amines to products that are mutagenic in the Ames test is shown in Figure 1. Hepatic S9 preparations derived from STZ-treated rats were markedly less efficient than the control animals in converting the aromatic amine 2-aminofluorene to mutagens. Concomitant administration of nicotinamide with STZ prevented the decrease in mutagenicity, and daily treatment of diabetic animals with insulin partially restored the mutagenic response to that obtained with activation systems from control animals. However, no significant changes were seen in the activation of 4-aminobiphenyl (Fig. 1). In marked contrast, the heterocyclic amine GluP-1 was more efficiently activated to mutagens by the
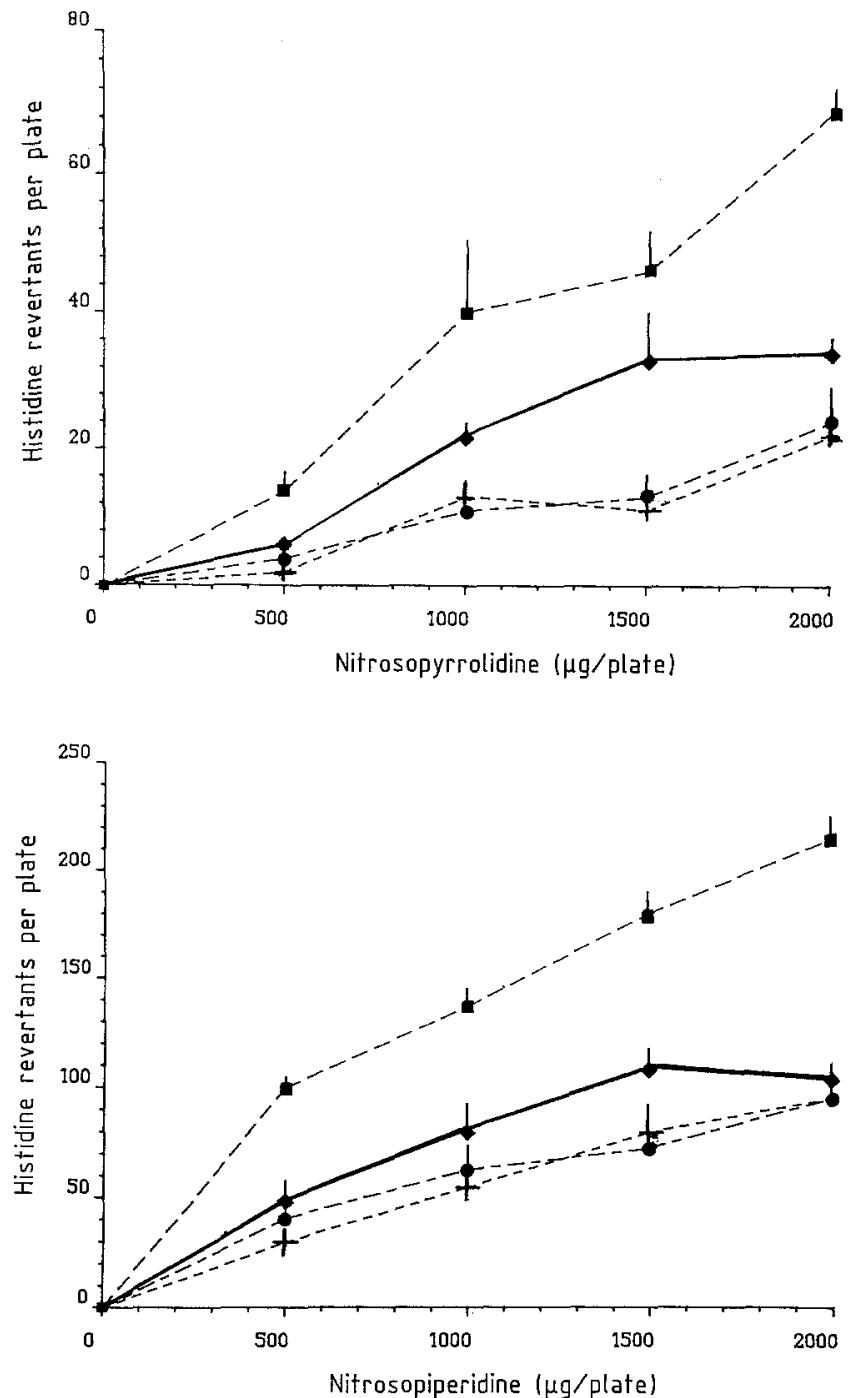

Fig. 3. The effect of streptozotocin-induced diabetes on the activation of nitrosamines to mutagens. The metabolic activation of nitrosopiperidine and nitrosopyrrolidine to mutagens in the Ames test was investigated using Salmonella typhimurium strain TA 1530 and employing $25 \%$ activation systems derived from control ( $)$, streptozotocin (更), streptozotocin + nicotinamide $(+)$ and streptozotocin + insulin $(\boldsymbol{O})$ treated animals. Results are presented as the mean $\pm S D$ of triplicates. The spontaneous reversion rate of $13 \pm 1$ has already been subtracted. The experiment was repeated with different, but similarly treated animals with the same results

streptozotocin-induced preparations when compared to the control preparations (Fig. 2). This enhancement was only partially preventable by nicotinamide and reversed by daily injection of insulin.

The polycyclic aromatic hydrocarbon benzo(a)pyrene was not activated by any of the $\mathrm{S} 9$ preparations (Table 1). A small increase over spontaneous reversion rate was evident, but never reached a doubling of this rate which is considered as a prerequisite for a positive mutagenic response. In contrast, 3-methylcholanthrene, another polycyclic aromatic hydrocarbon, was activated by all systems, to a similar degree (Table 1 ).

Treatment of rats with STZ markedly increased the activation of the two nitrosamines, namely nitrosopipe- 
ridine and nitrosopyrrolidine, to mutagens in the Ames test (Fig. 3). The increase was preventable by co-administration of nicotinamide, and it was successfully antagonised with insulin therapy.

\section{Discussion}

Epidemiological studies have suggested that diabetics may have a higher cancer incidence [17]. Studies in rats demonstrated that treatment with streptozotocin enhances the metabolism and hepatotoxicity of certain nitrosamines [13, 18], a major group of potent chemical carcinogens. Furthermore, recent studies indicate that the chemically-induced diabetic rats have increased capacity in converting some food carcinogens, generated during cooking, to mutagenic intermediates, apparently by modulating the levels of cytochrome P-450-proteins that are involved in their metabolic activation [12]. The present work forms an extension of these studies by investigating if STZ-induced diabetes modulates the activation pathways of prototypes of three major groups of environmental chemical carcinogens, namely aromatic amines, nitrosamines and polycyclic aromatic hydrocarbons.

A complication arising in studies where a diabetogenic agent has been administered to animals is the distinction between the effects of the agent per se and those of the resulting diabetes. Indeed Chawalet et al. [19] have reported differential effects of streptozotocinand alloxan-induced diabetes on the $p$-hydroxylation of aniline. Furthermore, murine hepatic glutathione transferase activity was stimulated by STZ through a mechanism independent of diabetes [20]. In order to discern the effects of diabetes from those of the chemical, a group of animals received, in addition to STZ, nicotinamide which counters the onset of diabetes [21].

Animals treated with STZ were less efficient in metabolically converting the aromatic amine 2-aminofluorene to mutagens when compared to control animals. Nicotinamide afforded protection against STZ indicating that this effect may be ascribed to the diabetic state. The bioactivation of another aromatic amine, namely 4-aminobiphenyl was not influenced by treatment of the rats with streptozotocin. In contrast, the activation of the heterocyclic amine Glu-P-1 was potentiated following induction of diabetes with STZ. Once again the increase in mutagenic response was prevented by concomitant administration of nicotinamide and was partially reversed by daily treatment with insulin indicating that the state of diabetes was responsible for the increased mutagenesis. These findings preclude generalisations as to the consequences of diabetes on the activation of even structurally related compounds since the effect is very much dependent upon the nature of the chemical. The activation of 2-aminofluorene, 4-aminobiphenyl and Glu-P-1 proceeds through $\mathrm{N}$-hydroxylation, yet the effect of diabetes is contrasting. However, different enzyme systems are involved in the activation process, such as the FAD-monooxygenase and different isozymes of cytochrome P-450 which may show different response to the state of diabetes.

When nitrosamines were investigated, diabetic animals were profoundly more effective in the activation of nitrosopiperidine and nitrosopyrrolidine. In both cases, the effect was preventable by nicotinamide and reversed to control levels by insulin therapy, thereby demonstrating that this effect was diabetes-induced. Recent studies have established that a specific isozyme of the cytochrome P-450 superfamily, namely cytochrome P450IIE (rabbit $\mathrm{LM}_{3 \mathrm{a}}$ ) inducible by alcohol, readily converts certain nitrosamines to their active intermediates(s) [22], and that the levels of this isozyme increase in STZ-induced and spontaneous diabetes [23, 24]. This provides a plausible explanation for the increased activation of the two nitrosamines observed in the present study. It has been suggested [13] that raised concentrations of circulating ketone bodies may be responsible for the induction of cytochrome P450 II E in both alcohol and STZ-treated animals, and this hypothesis is at present under investigation.

The mutagenic response elicited by the two polycyclic aromatic hydrocarbons benzo(a)pyrene and 3methycholanthrene was not affected by STZ treatment of the animals. The mutagenicity of the polycyclic aromatic hydrocarbons appears to be unaffected by STZinduced diabetes despite the fact that one of the enzyme systems involved in the activation of these carcinogens, namely cytochromes P-448 (P450 I) [5] is increased following induction of diabetes [12] and in their studies Peng et al. observed a decrease in the metabolism of benzo(a)pyrene. However, it must be stressed that the mutagenicity of this group of carcinogens is due to more than one metabolite [25], which are generated by different enzyme systems [26] that may show different response to diabetes. Peng et al. used microsomes in their studies while, in the mutagenicity studies presented in this paper S9 systems have been employed, i.e. microsomes + cytosolic fractions. Cytosolic enzymes can further metabolise microsome-generated metabolites so that they can modulate mutagenicity. Finally, in the studies of Peng et al. animals were diabetic for only 8 days in contrast to the 21 days adopted in the present study. The possibility that the severity and duration of diabetes may influence the cytochrome P-450 system cannot be excluded.

The present study clearly demonstrates that the activation of chemical carcinogens can be modulated by the diabetic state. The effect depends upon the nature of the carcinogen, and it is clear that diabetes has a selective rather than a "general" effect on the drug metabolising enzymes. Indeed, chemical induction of diabetes increases the levels of some cytochrome P-450 forms at the expense of others $[11,27]$, and even promotes the synthesis of novel forms $[10,24]$. 
Before the present observations are extrapolated to the in vivo situation a number of questions must be adequately addressed: a) Does diabetes perturb the metabolic pathways leading to deactivation, and what is the overall effect on the balance of activation/deactivation? Preliminary studies have revealed that diabetes may modulate a number of deactivation pathways [28, 29]; b) Repeated exposure to chemical carcinogens results in selective induction of the enzymes that catalyse their activation [5]. Are diabetic animals refractive or sensitive to induction by such agents? Increased sensitivity is likely to render the diabetic particularly susceptible to some carcinogens; c) Since the cytochrome P-450 enzyme system participates in the metabolism of many endogenous substrates including steroids, vitamins and fatty acids, is the turnover of these substrates affected by diabetes? The cytochrome P-450-dependent heaptic $7 \alpha$-hydroxylation of cholesterol, the rate limiting step in the biosynthesis of bile acids, is markedly increased by STZ and the effect is antagonised by insulin [30].

\section{References}

1. Nebert DW, Gonzalez FJ (1985) Cytochrome P-450 gene expression and regulation. Trends Pharm Sci 13:156-162

2. Ryan DE, Thomas PE, Reik LM, Levin W (1982) Purification, characterization and regulation of five rat hepatic cytochrome P450 isozymes. Xenobiotica 12: 727-744

3. Guengerich FP, Dannan GA, Wright ST, Martin MV, Keminsky LS (1982) Purification and characterization of liver microsomal cytochrome P450: electrophoretic, spectral, catalytic and immunochemical properties and inducibility of eight isozymes isolated from animals treated with phenobarbital or $\beta$-naphthoflavone. Biochemistry $26: 1406-1412$

4. Ioannides C, Lum PY, Parke DV (1984) Cytochrome P448 and the activation of toxic chemicals and carcinogens. Xenobiotica 14: 119-137

5. Ioannides C, Parke DV (1987) The cytochromes P-448 - a unique family of enzymes involved in chemical toxicity and carcinogenesis. Biochem Pharm 36: 4197-4207

6. Dixon RL, Hart LG, Rogers LA, Fouts JR (1963) The metabolism of drugs by liver microsomes from alloxan-diabetic rats: longterm diabetes. J Pharmacol Exp Ther 142: 312-317

7. Ackerman DM, Liebman KC (1977) Effect of experimental diabetes on drug metabolism in the rat. Drug Metab Dispos 5: 405-410

8. Reinke LA, Stohs SJ, Rosenberg H (1978) Altered activity of hepatic mixed-function monooxygenase enzymes in streptozotocininduced diabetic rats. Xenobiotica 8: 611-618

9. Rouer E, Leroux JP (1980) Liver microsomal cytochrome P450 related and monooxygenase activities in genetically hyperglycaemic (ob/ob and $\mathrm{db} / \mathrm{db}$ ) and lean streptozotocin-treated mice. Biochem Pharmacol 29: 1959-1962

10. Past MR, Cook DE (1982) Drug metabolism in a reconstituted system by diabetes-dependent hepatic cytochrome P450. Res Commun Chem Pathol Pharmacol 37: 81-90

11. Favreau LV, Schenkam JB (1987) Decrease in the levels of a constitutive cytochrome P450 (RLM5) in hepatic microsomes of diabetic rats. Biochem Biophys Res Commun 142: 623-630

12. Ioannides C, Bass SL, Ayrton AD, Trinick JE, Walker R, Flatt PR (in press) Streptozotocin-induced diabetes modulates the activation of chemical carcinogens. Chem Biol Interact 68:189-202

13. Peng R, Tennant P, Lorr A, Yang CS (1983) Alterations of microsomal monooxygenase system and carcinogen metabolism by streptozotocin-induced diabetes in rats. Carcinogenesis 4: 703-708

14. Maron DM, Ames BN (1983) Revised methods for the Salmonella mutagenicity test. Mutat Res 113:173-215

15. Stevens J (1971) Determination of glucose by an automatic analyser. Clin Chim Acta 38: 199-201

16. Ioannides C, Parke DV (1975) Mechanism of induction of hepatic microsomal drug metabolising enzymes by a series of barbiturates. J Pharm Pharmacol 27: 739-746

17. Volk W, Wellmann KF (1977) Cancer and diabetes. In: Volk BW, Wellman KF (eds) The diabetic pancreas. Plenum Press, New York, pp 311-316

18. Lorr NA, Miller KW, Haingsug RC, Yang CS (1984) Potentiation of the hepatotoxicity of $\mathrm{N}$-nitrosodimethylamine by fasting diabetes, acetone and isopropanol. Toxicol Appl Pharmacol 73: 423-431

19. Chawalet K, Sretanigsa P, Thithapandha A (1982) Comparative effects of diabetogenic agents on hepatic drug metabolism. Drug Metab Dispos 10: 81-86

20. Agius C, Gidari AS (1988) Effect of streptozotocin on the glutathione S-transferases of mouse liver cytosol. Biochem Pharmacol 34: 811-819

21. Schein P, Cooney D, Vernon L (1967) The use of nicotinamide to modify the toxicity of streptozotocin diabetes without loss of antitumor activity. Cancer Res 27: 2324-2332

22. Yang CS, Tu YY, Koop DR, Coon MJ (1985) Metabolism of nitrosamines by purified rabbit liver microsomal cytochrome $\mathrm{P} 450$ isozymes. Cancer Res 45: 1140-1145

23. Bellward GD, Chang T, Rodriques B, McNeill JH, Maines $\mathrm{S}$, Ryan D, Levin W, Thomas PE (1987) Hepatic cytochrome P450j induction in the BB spontaneously diabetic rat. The VII Symposium on Microsomes and Drug Oxidations. Adelaide, August 1987. Abstract P-60

24. Dong Z, Hong J, Ma Q, Li D, Bullock J, Gonzalez FJ, Park SS, Gelboin HV, Yang CS (1988) Mechanism of induction of cytochrome $\mathrm{P}-450_{\mathrm{ac}}(\mathrm{P}-450 \mathrm{j})$ in chemically induced and spontaneously diabetic rats. Arch Biochem Biophys 263: 29-35

25. Wood AW, Levin W, Lu AYH, Yagi H, Hernandez O, Jerina DM, Conney AH (1976) Metabolism of benzo(a)pyrene and benzo(a)pyrene derivatives to mutagenic products by highly purified microsomal enzymes. J Biol Chem 251: 4882-4890

26. Oesch F, Raphael D, Schind H, Glatt HR (1977) Species differences in activating and inactivating enzymes related to the control of mutagenic metabolites. Arch Toxicol 39: 97-108

27. Hietanen H, Rauramaa R, Laitinen M (1982) Effect of experimental diabetes on hepatic monooxygenases. In: Hietanen E, Laitinen M, Hanninen O (eds) Cytochrome P-450. Biochemistry, biophysics and environmental implications, developments in biochemistry, Vol 23. Elsevier Biomedical Press, Amsterdam, pp 781-784

28. Younes M, Schlichting R, Siegers CP (1980) Glutathione S-transferase activities in rat liver: effect of some factors influencing the metabolism of xenobiotics. Pharmacol Res Commun 12: 115-119

29. Rouer E, Mahu JL, Dansette P, Leroux JP (1981) UDP-glucuronyltransferase, epoxide hydrolase and glutathione S-transferase activities in the liver of diabetic mice. Biochim Biophys Acta 676: 274-277

30. Subbiah MTR, Yunker RL (1984) Cholesterol $7 \alpha$-hydroxylase of rat liver: An insulin sensitive enzyme. Biochem Biophys Res Commun 12: 896-902

Received: 25 August 1988

and in revised form: 12 December 1988

Dr. C. Ioannides

Department of Biochemistry

University of Surrey

Guildford, Surrey GU2 5XH

UK 